\title{
33. A New Model of the Excess Gibbs Energy of Mixing for a Regular Solution
}

\author{
By Yasushi KaKudA, Etsuo UCHIDA, and Naoya IMAI \\ Department of Mineral Resources Engineering, Waseda University, \\ 3-4-1 Ohkubo, Shinjuku-ku, Tokyo 169
}

(Communicated by Syun-iti Aкıмото, м. J. А., Dec. 12, 1994)

\begin{abstract}
The excess Gibbs energy of mixing for a regular solution can be derived systematically by defining the interaction energy for particle groups composed of closest neighbors at equivalent site. The internal energy of a system is regarded as the sum of the interaction energy for particle groups and the excess function is derived from the sum of the interaction energy change caused by the formations of particle groups.

In the present paper, we have derived the excess Gibbs energy of mixing for ternary and quaternary regular solutions with a closest packing. For a ternary regular solution, the molar excess Gibbs energy of mixing is given as follows,

$$
\begin{aligned}
\bar{G}^{e x} & =X_{A} X_{B}\left(X_{A} W_{A A B}+X_{B} W_{A B B}\right)+X_{B} X_{C}\left(X_{B} W_{B B C}+X_{C} W_{B C C}\right) \\
& +X_{C} X_{A}\left(X_{C} W_{C C A}+X_{A} W_{C A A}\right)+2 X_{A} X_{B} X_{C} W_{A B C},
\end{aligned}
$$

where $X_{i}$ is a mole fraction of the component $i$ and $W_{i j k}$ is an interaction parameter of [ijk] triplet. The following expressions for $n$-component systems are derived by the similar method by supposing interaction energy among $r$ particles. Some previously proposed models can be derived from our model.

$$
\begin{aligned}
& n=2, r=2: \bar{G}^{e x}=X_{A} X_{B} W_{A B} \quad \text { (symmetric binary regular solution model) } \\
& n=2, r=3: \bar{G}^{e x}=X_{A} X_{B}\left(X_{A} W_{A A B}+X_{B} W_{A B B}\right) \text { (asymmetric binary regular solution model) } \\
& n=3, r=2: \bar{G}^{e x}=X_{A} X_{B} W_{A B}+X_{B} X_{C} W_{B C}+X_{C} X_{A} W_{C A} \\
& \text { (symmetric ternary regular solution model) } \\
& n=3, r=3: \bar{G}^{e x}=X_{A} X_{B}\left(X_{A} W_{A A B}+X_{B} W_{A B B}\right)+X_{B} X_{C}\left(X_{B} W_{B B C}+X_{C} W_{B C C}\right) \\
& +X_{C} X_{A}\left(X_{C} W_{C C A}+X_{A} W_{C A A}\right)+2 X_{A} X_{B} X_{C} W_{A B C} \\
& \text { (new ternary model: stated above) } \\
& n=4, r=4: \bar{G}^{e x}=X_{A}{ }^{3} X_{B} W_{A A A B}+X_{B}{ }^{3} X_{A} W_{B B B A}+X_{A}{ }^{3} \mathrm{X}_{\mathrm{C}} \mathrm{W}_{\mathrm{AAAC}}+X_{C}{ }^{3} X_{A} W_{C C C A} \\
& +X_{A}{ }^{3} X_{D} W_{A A A D}+X_{D}{ }^{3} X_{A} W_{D D D A}+X_{B}^{3} X_{C} W_{B B B C}+X_{C}{ }^{3} X_{B} W_{C C C B} \\
& +X_{B}^{3} X_{D} W_{B B B D}+X_{D}{ }^{3} X_{B} W_{D D D B}+X_{C}{ }^{3} X_{D} W_{C C C D}+X_{D}{ }^{3} X_{C} W_{D D D C} \\
& +{ }^{3} / 2 X_{A}{ }^{2} X_{B}^{2} W_{A A B B}+3 / 2 X_{A}^{2} X_{C}^{2} W_{A A C C}+3 / 2 X_{A}^{2} X_{D}{ }^{2} W_{A A D D} \\
& +{ }^{3} /{ }_{2} X_{B}{ }^{2} X_{C}{ }^{2} W_{B B C C}+{ }^{3} /{ }_{2} X_{B}{ }^{2} X_{D}{ }^{2} W_{B B D D}+{ }^{3} /{ }_{2} X_{C}{ }^{2} X_{D}{ }^{2} W_{C C D D} \\
& +3 X_{A}^{2} X_{B} X_{C} W_{A A B C}+3 X_{A}^{2} X_{B} X_{D} W_{A A B D}+3 X_{A}^{2} X_{C} X_{D} W_{A A C D} \\
& +3 X_{B}^{2} X_{A} X_{C} W_{B B A C}+3 X_{B}^{2} X_{A} X_{D} W_{B B A D}+3 X_{B}^{2} X_{C} X_{D} W_{B B C D} \\
& +3 X_{C}^{2} X_{A} X_{B} W_{C C A B}+3 X_{C}^{2} X_{A} X_{D} W_{C C A D}+3 X_{C}^{2} X_{B} X_{D} W_{C C B D} \\
& +3 X_{D}^{2} X_{A} X_{B} W_{D D A B}+3 X_{D}^{2} X_{A} X_{C} W_{D D A C}+3 X_{D}^{2} X_{B} X_{C} W_{D D B C} \\
& +6 X_{A} X_{B} X_{C} X_{D} W_{A B C D} \text {. }
\end{aligned}
$$

(new quaternary model)

Key words: Regular solution; ternary system; quaternary system; thermodynamics; excess Gibbs energy.

Introduction. Regular solution, originally introduced by Hildebrand, ${ }^{1)}$ is one of the simplest solution models that have ever been derived. For a binary regular solution, 
symmetric regular solution moael is established by introducing the interaction energy of particle pairs (Guggenneim $\left.{ }^{2}\right)$, and then subregular solution model is derived as a higher expansion in the empirical method $\left(\mathrm{Hardy}^{33}\right)$. For ternary and quaternary regular solutions, algebraical solutions and empirical approaches are proposed by Anderson and Lindsley, ${ }^{4)}$ Barron, ${ }^{5)}$ Kohler $^{6)}$ and Wohl ${ }^{7,8)}$ but physical model have not been given yet.

The purpose of the present paper is to derive the excess Gibbs energy of mixing for ternary and quaternary regular solutions by introducing triplet and quadruplet, respectively, originally appeared in Guggenneim ${ }^{9)}$ for a binary system.

Symbols.
$\begin{array}{ll}G^{e x} & \text { Excess Gibos energy of mixing, } \\ \bar{G}^{e x} & \text { Molar excess Gibbs energy of mixing, }\end{array}$
[ijk] Triplet composed of the particles $i, j$ and $k$,
$N \quad$ Avogadro's number,
$N_{i} \quad$ Number of the particle $i$,
$N_{i j k} \quad$ Number of [ijk] triplets,
$<N_{i j k}>$ Average number of [ijk] triplets,
$n \quad$ Number of components in a system,
$n_{i} \quad$ Number of moles of the component $i$,
$r \quad$ Number of particles constituting a particle group,
$U \quad$ Internal energy,
$U^{e x} \quad$ Excess internal energy,
$W_{i j k} \quad$ Interaction parameter of [ijk] triplet,
$X_{i} \quad$ Mole fraction of the component $i ; \Sigma X_{i}=1$,
$z \quad$ Coordination number; $z=12$ for a ciosest packing,
$\varepsilon_{i j k} \quad$ Interaction energy of [ijk] triplet,
$\omega_{i j k} \quad$ Interaction energy change of formation of [ijk] triplet,
$\mu_{i}^{e x} \quad$ Excess chemical potential of the component $i$,
$\varphi^{i j k} \quad$ Probability of occurrence for [ijk] triplet.

Ternary regular solution. The authors derive the excess Gibbs energy of mixing for a ternary regular solution on the following assumptions:

(a) The total internal energy of the system is expressed as the sum of the interaction energy of all particle groups.

(b) Particles are of the same size and are distributed randomly without volume change at equivaient site.

(c) Only closest neignbors are subjected to interactions. Long range interactions such as electrostatic force can be ignored.

(d) Each particle is directly surrounded by other $z$ particles. The value of $z$ is 12 for a closest packing, but no assignment of $z$ value is needed.

From the assumption $(a)$, the internal energy of the system can be expressed as the sum of the internal energy of each triplet:

$$
\begin{aligned}
U & =\varepsilon_{A A A} N_{A A A}+\varepsilon_{B B B} N_{B B B}+\varepsilon_{C C C} N_{C C C}+\varepsilon_{A A B} N_{A A B}+\varepsilon_{A B B} N_{A B B} \\
& +\varepsilon_{B B C} N_{B B C}+\varepsilon_{B C C} N_{B C C}+\varepsilon_{C C A} N_{C C A}+\varepsilon_{C A A} N_{C A A}+\varepsilon_{A B C} N_{A B C} .
\end{aligned}
$$

As each particle is shared by $2 z$ triplets, shown in Fig. 1, we have the following relations between the number of triplets $\left(N_{i j k}\right)$ and the number of particles $\left(N_{i}\right)$ :

$$
\begin{aligned}
& 3 N_{A A A}+2 N_{A A B}+2 N_{C A A}+N_{A B B}+N_{C C A}+N_{A B C}=2 z N_{A} . \\
& 3 N_{B B B}+2 N_{A B B}+2 N_{B B C}+N_{A A B}+N_{B C C}+N_{A B C}=2 z N_{B}, \\
& 3 N_{C C C}+2 N_{C C A}+2 N_{B C C}+N_{C A A}+N_{B B C}+N_{A B C}=2 z N_{C} .
\end{aligned}
$$

The total number of triplets is given by the sum of the equations (2), (3) and (4): 


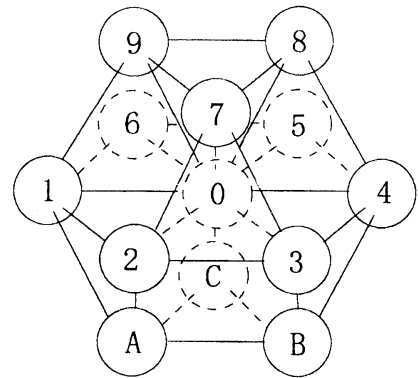

Fig. 1. A closest cubic packing composed of 24 triplets. [012], [016], [019], [01A], [023], [027], [02A], [034] [037], [03B], [045], [048], [04C], [056], [058], [05A], [069], [06C], [078], [079], [089], [0AC], [0BC] and [0BC], or composed of 8 quadruplets: [012A], [0237], [034B], [0458], [056C], [0169], [0ABC] and [0789]. A centered particle is surrounded by 12 closest neighbors.

$$
\begin{aligned}
& N_{A A A}+N_{B B B}+N_{C C C}+N_{A A B}+N_{B B C}+N_{C C A}+N_{A B B}+N_{B C C}+N_{C A A}+N_{A B C} \\
& \quad={ }_{2 / 3} z\left(N_{A}+N_{B}+N_{C}\right),
\end{aligned}
$$

Equations (1) to (4) give the following expression for the internal energy of the system:

$$
\begin{aligned}
U & ={ }^{2} / 3 z N_{A} \varepsilon_{A A A}+{ }^{2 / 3} z N_{B} \varepsilon_{B B B}+{ }^{2 / 3} z N_{C} \varepsilon_{C C C} \\
& +N_{A A B}\left\{\varepsilon_{A A B^{-1} / 3}\left(\varepsilon_{A A A}+\varepsilon_{A A A}+\varepsilon_{B B B}\right)\right\}+N_{A B B}\left\{\varepsilon_{A B B^{-1} / 3}\left(\varepsilon_{A A A}+\varepsilon_{B B B}+\varepsilon_{B B B}\right)\right\} \\
& +N_{B B C}\left\{\varepsilon_{B B C^{-1} / 3}\left(\varepsilon_{B B B}+\varepsilon_{B B B}+\varepsilon_{C C C}\right)\right\}+N_{B C C}\left\{\varepsilon_{B C C^{-1 / 3}}\left(\varepsilon_{B B B}+\varepsilon_{C C C}+\varepsilon_{C C C}\right)\right\} \\
& +N_{C C A}\left\{\varepsilon_{C C A^{-1 / 3}}\left(\varepsilon_{C C C}+\varepsilon_{C C C}+\varepsilon_{A A A}\right)\right\}+N_{C A A}\left\{\varepsilon_{C A A^{-1 / 3}}\left(\varepsilon_{C C C}+\varepsilon_{A A A}+\varepsilon_{A A A}\right)\right\} \\
& +N_{A B C}\left\{\varepsilon_{A B C^{-1} / 3}\left(\varepsilon_{A A A}+\varepsilon_{B B B}+\varepsilon_{C C C}\right)\right\} .
\end{aligned}
$$

Considering the mixing process as the formation of the triplets expressed by the following seven reactions,

$$
\begin{aligned}
& 1 / 3[\mathrm{AAA}]+{ }^{1} /{ }_{3}[\mathrm{AAA}]+{ }^{1} /{ }_{3}[\mathrm{BBB}]=[\mathrm{AAB}],{ }^{1} /{ }_{3}[\mathrm{AAA}]+{ }^{1 / 3}[\mathrm{BBB}]+1 / 3[\mathrm{BBB}]=[\mathrm{ABB}], \\
& 1 / 3[\mathrm{BBB}]+1 / 3[\mathrm{BBB}]+{ }^{1} /{ }_{3}[\mathrm{CCC}]=[\mathrm{BBC}], 1 /{ }_{3}[\mathrm{BBB}]+{ }^{1} /{ }_{3}[\mathrm{CCC}]+{ }^{1} /{ }_{3}[\mathrm{CCC}]=[\mathrm{BCC}] \text {, } \\
& 1 /{ }_{3}[\mathrm{CCC}]+1 / \frac{1}{3}[\mathrm{CCC}]+1 /{ }_{3}[\mathrm{AAA}]=[\mathrm{CCA}], 1 / 3{ }_{3}[\mathrm{CCC}]+1 /{ }_{3}[\mathrm{AAA}]+1 /{ }_{3}[\mathrm{AAA}]=[\mathrm{CAA}] \text {, } \\
& \text { and } 1 /{ }_{3}[\mathrm{AAA}]+{ }^{1} /{ }_{3}[\mathrm{BBB}]+{ }^{1} /{ }_{3}[\mathrm{CCC}]=[\mathrm{ABC}] \text {, }
\end{aligned}
$$

the interaction energy changes of formation of [ijk] triplets in equation (7) are defined as,

$$
\begin{aligned}
\omega_{A A B} & =\varepsilon_{A A A^{-1} / 3}\left(\varepsilon_{A A A}+\varepsilon_{A A A}+\varepsilon_{B B B}\right), \omega_{A B B}=\varepsilon_{A A A^{-1} / 3}\left(\varepsilon_{A A A}+\varepsilon_{B B B}+\varepsilon_{B B B}\right), \\
\omega_{B B C} & =\varepsilon_{B B B^{-1} / 3}\left(\varepsilon_{B B B}+\varepsilon_{B B B}+\varepsilon_{C C C}\right), \omega_{B C C}=\varepsilon_{B B B^{-1} / 3}\left(\varepsilon_{B B B}+\varepsilon_{C C C}+\varepsilon_{C C C}\right), \\
\omega_{C C A} & =\varepsilon_{C C C^{-1} / 3}\left(\varepsilon_{C C C}+\varepsilon_{C C C}+\varepsilon_{A A A}\right), \omega_{C A A}=\varepsilon_{C C C^{-1} / 3}\left(\varepsilon_{C C C}+\varepsilon_{A A A}+\varepsilon_{A A A}\right), \\
\text { and } \omega_{A B C} & =\varepsilon_{A A A^{-1} / 3}\left(\varepsilon_{A A A}+\varepsilon_{B B B}+\varepsilon_{C C C}\right), \text { respectively. }
\end{aligned}
$$

Therefore, the internal energy can be rewritten using equation (8) as

$$
\begin{aligned}
& U={ }^{1 / 3} z N_{A} \varepsilon_{A A A}+{ }^{2} / 3 z N_{b} \varepsilon_{B B B}+2 / 3 z N_{C} \varepsilon_{C C C}+N_{A A B} \omega_{A A B}+N_{A B B} \omega_{A B B}+N_{B B C} \omega_{B B C} \\
& +N_{B C C} \omega_{B C C}+N_{C C A} \omega_{C C A}+N_{C A A} \omega_{C A A}+N_{A B C} \omega_{A B C} \text {, }
\end{aligned}
$$

and then the excess internal energy is given by

$$
\begin{aligned}
U^{e x} & =N_{A A B} \omega_{A A B}+N_{A B B} \omega_{A B B}+N_{B B C} \omega_{B B C}+N_{B C C} \omega_{B C C} \\
& +N_{C C A} \omega_{C C A}+N_{C A A} \omega_{C A A}+N_{A B C} \omega_{A B C} .
\end{aligned}
$$

Based on the assumption $(b)$, the Gibbs energy can be considered to be the same as the internal energy. Therefore, equation (10) will be rewritten as

$$
\begin{aligned}
G^{e x} & =N_{A A B} \omega_{A A B}+N_{A B B} \omega_{A B B}+N_{B B C} \omega_{B B C}+N_{B C C} \omega_{B C C} \\
& +N_{C C A} \omega_{C C A}+N_{C A A} \omega_{C A A}+N_{A B C} \omega_{A B C} .
\end{aligned}
$$

From the random distribution of particles in the assumption $(b)$, the probability of occurrence for [ijk] triplet is defined as follows; 


$$
\begin{aligned}
\varphi_{A A B} & =\frac{3 N_{A}^{2} N_{B}}{\left(N_{A}+N_{B}+N_{C}\right)^{3}}, \varphi_{A B B}=\frac{3 N_{A} N_{B}^{2}}{\left(N_{A}+N_{B}+N_{C}\right)^{3}}, \varphi_{B B C}=\frac{3 N_{B}^{2} N_{C}}{\left(N_{A}+N_{B}+N_{C}\right)^{3}}, \\
\varphi_{B C C} & =\frac{3 N_{B} N_{C}^{2}}{\left(N_{A}+N_{B}+N_{C}\right)^{3}}, \varphi_{C C A}=\frac{3 N_{C}^{2} N_{A}}{\left(N_{A}+N_{B}+N_{C}\right)^{3}}, \varphi_{C A A}=\frac{3 N_{C} N_{A}^{2}}{\left(N_{A}+N_{B}+N_{C}\right)^{3}}, \\
\text { and } \varphi_{A B C} & =\frac{6 N_{A} N_{B} N_{C}}{\left(N_{A}+N_{B}+N_{C}\right)^{3}} .
\end{aligned}
$$

Equations (5) and (12) give the average number of triplets in the system as

$$
\begin{aligned}
& <N_{A A B}>=\frac{2 z N_{A}{ }^{2} N_{B}}{\left(N_{A}+N_{B}+N_{C}\right)^{2}}=\frac{2 z n_{A}{ }^{2} n_{B}}{\left(n_{A}+n_{B}+n_{C}\right)^{2}} N, \quad<N_{A B B}>=\frac{2 z N_{A} N_{B}{ }^{2}}{\left(N_{A}+N_{B}+N_{C}\right)^{2}}=\frac{2 z n_{A} n_{B}{ }^{2}}{\left(n_{A}+n_{B}+n_{C}\right)^{2}} N, \\
& <N_{B B C}>=\frac{2 z N_{B}{ }^{2} N_{C}}{\left(N_{A}+N_{B}+N_{C}\right)^{2}}=\frac{2 z n_{B} n_{C}}{\left(n_{A}+n_{B}+n_{C}\right)^{2}} N, \quad<N_{B C C}>=\frac{2 z N_{B} N_{C}{ }^{2}}{\left(N_{A}+N_{B}+N_{C}\right)^{2}}=\frac{2 z n_{B} n_{C}{ }^{2}}{\left(n_{A}+n_{B}+n_{C}\right)^{2}} N, \\
& <N_{C C A}>=\frac{2 z N_{C}{ }^{2} N_{A}}{\left(N_{A}+N_{B}+N_{C}\right)^{2}}=\frac{2 z n_{C} n_{A}}{\left(n_{A}+n_{B}+n_{C}\right)^{2}} N, \quad<N_{C A A}>=\frac{2 z N_{B} N_{A}^{2}}{\left(N_{A}+N_{B}+N_{C}\right)^{2}}=\frac{2 z n_{C} n_{A}{ }^{2}}{\left(n_{A}+n_{B}+n_{C}\right)^{2}} N, \\
& \text { and }<N_{A B C}>=\frac{4 z N_{A} N_{B} N_{C}}{\left(N_{A}+N_{B}+N_{C}\right)^{2}}=\frac{4 z n_{A} n_{B} n_{C}}{\left(n_{A}+n_{B}+n_{C}\right)^{2}} N .
\end{aligned}
$$

Substituting $N_{i j k}$ in equation (11) with $<N_{i j k}>$ in equation (13), the excess Gibbs energy of. mixing is rewritten as

$$
\begin{aligned}
G^{e x}= & 2 z \frac{n_{A}{ }^{2} n_{B}}{\left(n_{A}+n_{B}+n_{C}\right)^{2}} \omega_{A A B} N+2 z \frac{n_{A} n_{B}{ }^{2}}{\left(n_{A}+n_{B}+n_{C}\right)^{2}} \omega_{A B B} \boldsymbol{N}+2 z \frac{n_{B}{ }^{2} n_{C}}{\left(n_{A}+n_{B}+n_{C}\right)} \omega_{B B C} \boldsymbol{N} \\
& +2 z \frac{n_{B}{ }^{2} n_{C}{ }^{2}}{\left(n_{A}+n_{B}+n_{C}\right)^{2}} \omega_{B C C} \boldsymbol{N}+2 z \frac{n_{C}{ }^{2} n_{A}}{\left(n_{A}+n_{B}+n_{C}\right)^{2}} \omega_{C C A} \boldsymbol{N}+2 z \frac{n_{C} n_{A}{ }^{2}}{\left(n_{A}+n_{B}+n_{C}\right)} \omega_{C A A} \boldsymbol{N} \\
& +4 z \frac{n_{A} n_{B} n_{C}}{\left(n_{A}+n_{B}+n_{C}\right)} \omega_{A B C} \boldsymbol{N} .
\end{aligned}
$$

Rewriting on the molar basis gives:

$$
\begin{aligned}
\bar{G}^{e x} & =2 z X_{A} X_{B}\left(X_{A} \omega_{A A B}+X_{B} \omega_{A B B}\right) N+2 z X_{B} X_{C}\left(X_{B} \omega_{B B C}+X_{C} \omega_{B C C}\right) N+2 z X_{C} X_{A}\left(X_{C} \omega_{C C A}\right. \\
& \left.+X_{A} \omega_{C A A}\right) N+4 z X_{A} X_{B} X_{C} \omega_{A B C} N .
\end{aligned}
$$

As each particle is shared by $2 z$ triplets, interaction parameter is defined as

$$
W_{i j k}=2 z \omega_{i j k} N \text {. }
$$

Using equation (16), the molar excess Gibbs energy of mixing can be rewritten in the following form:

$$
\begin{gathered}
\bar{G}^{e x}=X_{A} X_{B}\left(X_{A} W_{A A B}+X_{B} W_{A B B}\right)+X_{B} X_{C}\left(X_{B} W_{B B C}+X_{C} W_{B C C}\right) \\
\quad+X_{C} X_{A}\left(X_{C} W_{C C A}+X_{A} W_{C A A}\right)+2 X_{A} X_{B} X_{C} W_{A B C} .
\end{gathered}
$$

The excess chemical potential of the component $i$, derived by partial differentiation of $G^{e x}$ with respect to $n_{i}$, is given by

$$
\begin{aligned}
& \mu_{A}^{e x}=2 X_{A} X_{B}\left(1-X_{A}\right) W_{A A B}+X_{B}^{2}\left(1-2 X_{A}\right) W_{A B B}+X_{C}^{2}\left(1-2 X_{A}\right) W_{C C A}+2 X_{C} X_{A}\left(1-X_{A}\right) W_{C A A} \\
& -2 X_{B}^{2} X_{C} W_{B B C}-2 X_{B} X_{C}{ }^{2} W_{B C C}+2 X_{B} X_{C}\left(1-2 X_{A}\right) W_{A B C} \\
& \mu_{B}^{e x}=2 X_{B} X_{C}\left(1-X_{B}\right) W_{B B C}+X_{C}^{2}\left(1-2 X_{B}\right) W_{B C C}+X_{A}^{2}\left(1-2 X_{B}\right) W_{A A B}+2 X_{A} X_{B}\left(1-X_{B}\right) W_{A B B} \\
& -2 X_{C}^{2} X_{A} W_{C C A}-2 X_{C} X_{A}{ }^{2} W_{C A A}+2 X_{C} X_{A}\left(1-2 X_{B}\right) W_{A B C}, \\
& \text { and } \mu_{C}^{e x}=2 X_{C} X_{A}\left(1-X_{C}\right) W_{C A A}+X_{A}{ }^{2}\left(1-2 X_{C}\right) W_{C A A}+X_{B}^{2}\left(1-2 X_{C}\right) W_{B B C}+2 X_{B} X_{C}\left(1-X_{C}\right) W_{B C C} \\
& -2 X_{A}^{2} X_{B} W_{A A B}-2 X_{A} X_{B}{ }^{2} W_{A B B}+2 X_{A} X_{B}\left(1-2 X_{C}\right) W_{A B C} .
\end{aligned}
$$

Binary regular solution. For a binary solution, by substituting zero for $X_{C}$ in equation (17), the following expression is obtained: 


$$
\bar{G}^{e x}=X_{A} X_{B}\left(X_{A} W_{A A B}+X_{B} W_{A B B}\right) .
$$

Quaternary regular solution. In order to describe $\bar{G}^{e x}$ for a quaternary regular solution, we need to introduce quadruplet, instead of triplet, that forms a tetrahedron. As each particle is shared by 2/3z quadruplets as shown in Fig. 1, interaction parameter is defined as

$$
W_{i j k l}={ }^{2} / 3 z \omega_{i j k l} N .
$$

Then the following expression is obtained for a quaternary system by the same procedure as for a ternary solution:

$$
\begin{aligned}
\bar{G}^{e x} & =X_{A}{ }^{3} X_{B} W_{A A A B}+X_{B}{ }^{3} X_{A} W_{B B B A}+X_{A}{ }^{3} X_{C} W_{A A A C}+X_{C}{ }^{3} X_{A} W_{C C C A}+X_{A}{ }^{3} X_{D} W_{A A A D} \\
& +X_{D}{ }^{3} X_{A} W_{D D D A}+X_{B}{ }^{3} X_{C} W_{B B B C}+X_{C}{ }^{3} X_{B} W_{C C C B}+X_{B}{ }^{3} X_{D} W_{B B B D}+X_{D}{ }^{3} X_{B} W_{D D D B} \\
& +X_{C}{ }^{3} X_{D} W_{C C C D}+X_{D}{ }^{3} X_{C} W_{D D D C} \\
& +{ }^{3}{ }_{2} X_{A}{ }^{2} X_{B}{ }^{2} W_{A A B B}+{ }^{3} /{ }_{2} X_{A}{ }^{2} X_{C}{ }^{2} W_{A A C C}+{ }^{3} /{ }_{2} X_{A}{ }^{2} X_{D}{ }^{2} W_{A A D D}+{ }^{3}{ }_{2} X_{B}{ }^{2} X_{C}{ }^{2} W_{B B C C} \\
& +{ }_{2}{ }_{2} X_{B}{ }^{2} X_{D}{ }^{2} W_{B B D D}+{ }^{3} /{ }_{2} X_{C}{ }^{2} X_{D}{ }^{2} W_{C C D D} \\
& +3 X_{A}^{2} X_{B} X_{C} W_{A A B C}+3 X_{A}{ }^{2} X_{B} X_{D} W_{A A B D}+3 X_{A}{ }^{2} X_{C} X_{D} W_{A A C D}+3 X_{B}{ }^{2} X_{A} X_{C} W_{B B A C} \\
& +3 X_{B}{ }^{2} X_{A} X_{D} W_{B B A D}+3 X_{B}{ }^{2} X_{C} X_{D} W_{B B C D}+3 X_{C}{ }^{2} X_{A} X_{B} W_{C C A B}+3 X_{C}{ }^{2} X_{A} X_{D} W_{C C A D} \\
& +3 X_{C}^{2} X_{B} X_{D} W_{C C B D}+3 X_{D}{ }^{2} X_{A} X_{B} W_{D D A B}+3 X_{D}{ }^{2} X_{A} X_{C} W_{D D A C}+3 X_{D}{ }^{2} X_{B} X_{C} W_{D D B C} \\
& +6 X_{A} X_{B} X_{C} X_{D} W_{A B C D}
\end{aligned}
$$

Margules model. The Margules expansion, ${ }^{10)}$ essentially the same as the Guggenheim expansion, ${ }^{11)}$ is often applied to the expression of the excess Gibbs energy of mixing.

$$
\begin{aligned}
\bar{G}^{e x}=A+B X_{2}+C X_{2}{ }^{2}+D X_{2}{ }^{3}+\cdots & : \text { Binary system } \\
\bar{G}^{e x}=A+B X_{2}+C X_{3}+D X_{2}{ }^{2}+E X_{2} X_{3}+F X_{3}{ }^{2}+G X_{2}{ }^{3}+H X_{2} X_{3}{ }^{2}+I X_{2}{ }^{2} X_{3}+J X_{3}{ }^{3}+\cdots & \text { : Ternary system }
\end{aligned}
$$

The degree of Margules expansion corresponds to the number of interactive closest neighbors $(r)$ of our model. The Margules expansion to the second-degree terms gives the same expression as equation (21) for a binary regular solution.

$$
\bar{G}^{e x}=X_{1} X_{2}\left(X_{1} W_{12}+X_{2} W_{21}\right) .
$$

For a ternary regular solution, the similar expression to our model can be derived from equation (26) to the third-degree terms:

$$
\begin{aligned}
\bar{G}^{e x} & =X_{1} X_{2}\left(X_{1} W_{12}+X_{2} W_{21}\right)+X_{2} X_{3}\left(X_{2} W_{23}+X_{3} W_{32}\right) \\
& +X_{3} X_{1}\left(X_{3} W_{31}+X_{1} W_{13}\right)+X_{1} X_{2} X_{3} W_{123} .
\end{aligned}
$$

The difference between equations (17) and (28),

$$
W_{123}=2 W_{A B C},
$$

is caused by the fact that each coefficient in Margules model is just a fitting parameter, whereas each interaction parameter in our model signifies the molar excess interaction energy change of formation of [ijk] triplet.

Thompson's model. Symmetric ternary regular solution model, known as Thompson's model, ${ }^{12)}$ can be algebraically derived from the Margules expansion or empirically deduced from symmetric binary regular solution model.

By decomposing triplets into pairs, our model results in symmetric ternary regular solution model. As each pair is shared by four triplets, interaction energy of triplet can be rewritten using that of pair $\left(\varepsilon_{i j}\right)$ : 


$$
\begin{aligned}
& \varepsilon_{A A B}=1 / 4\left(\varepsilon_{A A}+\varepsilon_{A B}+\varepsilon_{A B}\right), \\
& \varepsilon_{B B C}=1 /{ }_{4}\left(\varepsilon_{B B}+\varepsilon_{B C}+\varepsilon_{B C}\right), \\
& \varepsilon_{C C A}=1 / 4\left(\varepsilon_{C C}+\varepsilon_{C A}+\varepsilon_{C A}\right), \\
& \varepsilon_{A B C}=1 / 4\left(\varepsilon_{A A}+\varepsilon_{B C}+\varepsilon_{C A}\right),
\end{aligned}
$$$$
\varepsilon_{A B B}=1 / 4\left(\varepsilon_{B B}+\varepsilon_{A B}+\varepsilon_{A B}\right),
$$$$
\varepsilon_{B C C}=1 / 4\left(\varepsilon_{C C}+\varepsilon_{B C}+\varepsilon_{B C}\right) \text {, }
$$$$
\varepsilon_{C A A}=1 / 4\left(\varepsilon_{A A}+\varepsilon_{C A}+\varepsilon_{C A}\right) \text {, }
$$

By defining the excess interaction energy of pair $\left(\omega_{i j}\right)$ in the same way as that of triplet;

$$
\omega_{A B}=\varepsilon_{A B^{-}}{ }^{-1 / 2}\left(\varepsilon_{A A}+\varepsilon_{B B}\right), \omega_{B C}=\varepsilon_{B C^{-1 / 2}}\left(\varepsilon_{B B}+\varepsilon_{C C}\right) \text {, and } \omega_{C A}=\varepsilon_{C A^{-1} / 2}\left(\varepsilon_{C C}+\varepsilon_{A A}\right) \text {, }
$$

the excess interaction energy of triplet can be expressed with that of pair as follows;

$$
\omega_{A A B}=\omega_{A B B}=1 / 2 \omega_{A B}, \quad \omega_{B B C}=\omega_{B C C}=1 / 2 \omega_{B C}, \quad \omega_{C C A}=\omega_{C A A}=1 / 2 \omega_{C A},
$$

and $\omega_{A B C}=1 / 4\left(\omega_{A B}+\omega_{B C}+\omega_{C A}\right)$.

Substituting equation (32) into (16), interaction parameter of triplet $\left(W_{i j k}\right)$ can be rewritten using that of pair $\left(W_{i j}\right)$ :

$$
\begin{array}{ll}
W_{A A B}=W_{A B B}=z \omega_{A B} N\left(\equiv W_{A B}\right), & W_{B B C}=W_{B C C}=z \omega_{B C} N\left(\equiv W_{B C}\right), \\
W_{C C A}=W_{C A A}=z \omega_{C A} N\left(\equiv W_{C A}\right), & W_{A B C}=1 / 2\left(W_{A B}+W_{B C}+W_{C A}\right) .
\end{array}
$$

This is a consequence that each particle is shared by $z$ pairs and then each pair by two triplets. By substituting equation (33) into (17), Thompson's model is obtained:

$$
\bar{G}^{e x}=X_{A} X_{B} W_{A B}+X_{B} X_{C} W_{B C}+X_{C} X_{A} W_{C A} .
$$

Substituting zero for $X_{C}$, equation (34) results in symmetric binary solution model:

$$
\bar{G}^{e x}=X_{A} X_{B} W_{A B} .
$$

The following model proposed by Thompson ${ }^{12)}$ as asymmetric ternary regular solution model is not consistent with our model given by equation (17).

$$
\bar{G}^{e x}=X_{A} X_{B}\left(X_{A} W_{A B}+X_{B} W_{B A}\right)+X_{B} X_{C}\left(X_{B} W_{B C}+X_{C} W_{C B}\right)+X_{C} X_{A}\left(X_{C} W_{C A}+X_{A} W_{A C}\right) .
$$

Remarks. The present regular solution model still contains the contradiction in the assumption such that random mixing takes place in spite of non-zero excess energy of mixing. However, the present model has advantage that the coefficients in our model have physical meanings (interaction parameter) and our model can derive several previous models.

Acknowledgments. The authors would like to thank Professor S. Banno of Kyoto University and Dr. Y. Ogasawara of Waseda University for helpful suggestions and encouragement during the course of this study. Thanks are also due to Prof. Emeritus S. Akimoto, M. J. A. for the communication to the Japan Academy.

\section{References}

1) Hildebrand, J. H. (1929): J. Am. Chem. Soc., 51, 66-80.

2) Guggenheim, E. A. (1935): Proc. Roy. Soc. A., 148, 304-312.

3) Hardy, H. K. (1953): Acta Metal., 1, 202-209.

4) Anderson, D. J., and Lindsley, D. H. (1981): Geochem. Cosmochim. Acta, 45, 847-853.

5) Barron, L. M. (1976): Geochem. J., 10, 137-144.

6) Kohler, F. (1960): Monatsh. Chem., 91, 738-740.

7) Wohl, K. (1946): Trans. Am. Inst. Chem. Eng., 42, 215-249.

8) - (1953): ibid., 49, 218-219.

9) Guggenneim, E. A. (1952): Mixtures, Clarendon Press, Oxford.

10) Margules, M. (1895): Wien Akad., 104, 1243-1278.

11) Guggenneim, E. A. (1937): Trans. Faraday Soc., 33, 151-159.

12) Thompson, J. B. Jr. (1967): Researches in Geochemistry, 2, 340-361. 